\title{
Interval Approaches for Uncertain Reasoning
}

\author{
Y.Y. Yao ${ }^{1}$ and S.K.M. Wong ${ }^{2}$ \\ ${ }^{1}$ Department of Computer Science, Lakehead University \\ Thunder Bay, Ontario, Canada P7B 5E1 \\ E-mail: yyao@flash.lakeheadu.ca \\ 2 Department of Computer Science, University of Regina \\ Regina, Saskatchewan, Canada S4S 0A2 \\ E-mail: wong@cs.uregina.ca
}

\begin{abstract}
This paper presents a framework for reasoning using intervals. Two interpretations of intervals are examined, one treats intervals as bounds of a truth evaluation function, and the other treats end points of intervals as two truth evaluation functions. They lead to two different reasoning approaches, one is based on interval computations, and the other is based on interval structures. A number of interval based reasoning methods are reviewed and compared within the proposed framework.
\end{abstract}

\section{Introduction}

Traditionally, inference is performed using a single-valued truth evaluation function. A proposition takes a single element from a set of truth values as its truth value. For example, in classical logic, a proposition is either true or false. In manyvalued logic systems such as fuzzy logic, a proposition takes a number between 0 and 1 as its truth value. In probabilistic logic, the truth value of a proposition is also a number between 0 and 1 , but is associated with a probabilistic interpretation. These approaches have many practical problems [11]. It may be unrealistic to expect an expert to provide a precise and reliable truth evaluation function. The maintenance of consistency using single-valued functions may be a difficult task. To resolve some of these problems, various proposals have been suggested using intervals as truth values $[2,3,10-12,15,17-19]$. These studies have resulted in many interval based tools for uncertain reasoning, such as incidence calculus [3,20], interval structures [14], belief and plausibility functions [12], necessity and possibility functions [4], and interval fuzzy reasoning [19].

There are at least two interpretations regarding the physical meanings of interval-valued truth. Intervals may be interpreted as bounds of an unknown single-valued truth evaluation function. Alternatively, the end points of intervals can be interpreted as two truth evaluation functions, each is a single-valued evaluation function. The main objective of this paper is to outline a framework for interval based reasoning and to study some basic issues. With respect to the two interpretations of interval-valued truth, we introduce the corresponding reasoning methods, one is based on interval computations, and the other is based on interval structures. 


\section{Interval based Reasoning}

We consider three basic notions, propositional formulas, truth values, and evaluation functions, in a reasoning system. Given a particular situation, typically we have a finite and non-empty set of primitive propositions $\Phi$, which can be regarded as a set of basic events [5]. The set $L(\Phi)$ of propositional formulas generated by $\Phi$ is the closure of $\Phi$ under negation $(\neg)$ and conjunction $(\wedge)$. For convenience, we define two special formulas $\top$ and $\perp$. Other connectives such as the disjunction $(\vee)$, implication $(\rightarrow)$, and equivalence $(\leftrightarrow)$ can be defined in terms of negation and conjunction. Let $V$ denote a set of truth values. A truth evaluation function $v: L(\Phi) \longrightarrow V$ is a mapping from the set of propositional formulas to the set of truth values. It provides interpretations of elements of $L(\Phi)$ in terms of elements of $V$. For this reason, an evaluation function is also referred to as a meaning function. Usually, certain operations are defined on the set of truth values in order to interpret the logical connectives in $L(\Phi)$. An evaluation function must also satisfy a set of axioms. The following examples are special reasoning systems.

Example 1. Two-valued propositional logic. In the classical two-valued propositional logic, the set of truth values is the two-element Boolean algebra $B_{2}=$ $\{T, F\}$. An evaluation function $v$ from $L(\Phi)$ to $B_{2}$ should satisfy the axioms:

$$
\begin{array}{ll}
\text { (c1) } & v(\perp)=F, \quad v(\top)=T, \\
\text { (c2) } & v(\neg \phi)=\neg v(\phi), \\
(\mathrm{c} 3) & v(\phi \wedge \psi)=v(\phi) \wedge v(\psi), \\
(\mathrm{c} 4) & v(\phi \vee \psi)=v(\phi) \vee v(\psi) .
\end{array}
$$

Note that the same set of symbols is used for both operations on $L(\Phi)$ and $B_{2}$. These axioms are not a minimal set.

Example 2. Min-max fuzzy logic. In the min-max fuzzy system ([0,1], 1-(), min, $\max )$ proposed by Zadeh [21], the set of truth values is the unit interval $[0,1]$ with lattice operations min and max. In this system, an evaluation function $f v$ satisfies the axioms:

$$
\begin{array}{ll}
\text { (f1) } & f v(\perp)=0, \quad f v(\top)=1, \\
\text { (f2) } & f v(\neg \phi)=1-f v(\phi), \\
\text { (f3) } & f v(\phi \wedge \psi)=\min (f v(\phi), f v(\psi)), \\
\text { (f4) } & f v(\phi \vee \psi)=\max (f v(\phi), f v(\psi)) .
\end{array}
$$

In general, one may interpret fuzzy logic connectives using triangular norms (t-norms) and conorms (t-conorms) [7].

Example 3. Probabilistic logic. In probabilistic logic, the set of truth values is the unit interval $[0,1]$ with arithmetic operations, such as addition and multiplication. An evaluation function $P$ in this system must satisfy the axioms:

$$
P(\perp)=0, \quad P(\top)=1,
$$




$$
\begin{array}{ll}
\text { (p2) } & P(\neg \phi)=1-P(\phi), \\
\text { (p3) } & P(\phi \wedge \psi)=P(\phi)+P(\psi)-P(\phi \vee \psi), \\
\text { (p4) } & P(\phi \vee \psi)=P(\phi)+P(\psi)-P(\phi \wedge \psi) .
\end{array}
$$

Axiom (p3) is equivalent to (p4), and (p2) can be derived from other axioms.

Example 4. Incidence calculus - possible worlds semantics of logic. Let $W$ denote a set of possible worlds. The power set $2^{W}$, equipped with set-theoretic operations $\sim, \cap$, and $\cup$, is used as the set of truth values $[3,17,20]$. An evaluation function $i$ associates a proposition in $L(\Phi)$ with a subset of $W$, and must satisfy the axioms:

(i1) $\quad i(\perp)=\emptyset, \quad i(\top)=W$

(i2) $\quad i(\neg \phi)=\sim i(\phi)$,

(i3) $\quad i(\phi \wedge \psi)=i(\phi) \cap i(\psi)$,

(i4) $\quad i(\phi \vee \psi)=i(\phi) \cup i(\psi)$.

The set $i(\phi)$ may be interpreted as the subset of possible worlds in which $\phi$ is true. The evaluation function $i$ is referred to as an incidence mapping [3].

The above examples cover both numeric and non-numeric evaluation functions. A common feature of these systems is that the set of truth values $V$ is equipped with an order relation. Suppose $\preceq$ is an order relation on $V$, for two elements $a, b \in V$ such that $a \preceq b$, we define a closed interval:

$$
[a, b]=\{x \mid a \preceq x \preceq b\} .
$$

That is, the interval $[a, b]$ consists of all elements between two end points $a$ and $b$. Typically, the system $(V, \preceq)$ is a lattice. Let $I(V)$ denote the set of all closed intervals on $V$. We assume that $V$ has a universal maximum element, denoted by 1 , and a universal minimum element, denoted by 0 . Clearly, $[0,1]$ belongs to $I(V)$. With the notion of intervals, one may consider an evaluation from $L(\Phi)$ to $I(V)$. This provides an interval extension of the proposed framework.

The introduction of the interval extension is motivated by practical needs. In many situations, it may be difficult to specify precisely and consistently an evaluation function from $L(\Phi)$ to $V$ satisfying certain axioms. To resolve this problem, one may use a pair of lower and upper bounds which define the range of the actual evaluation function. In this case, intervals are interpreted as bounds of an unknown evaluation function. Formally, we can introduce a pair of evaluations $v_{*}, v^{*}: L(\Phi) \longrightarrow V$ such that $v_{*}(\phi) \preceq v(\phi) \preceq v^{*}(\phi)$ for all $\phi \in L(\Phi)$, where $v$ denotes the actual evaluation function. In general, the lower and upper evaluations $v_{*}$ and $v^{*}$ belong to a class of evaluations that are different from those of the class containing $v$, denoted by $C_{v}$. The evaluations $v_{*}$ and $v^{*}$ may be interpreted as constraints that characterize the following subset of evaluations in $C_{v}$ :

$$
I C\left(v_{*}, v^{*}\right)=\left\{u \in C_{v} \mid v_{*}(\phi) \preceq u(\phi) \preceq v^{*}(\phi) \text { for all } \phi \in L(\Phi)\right\} .
$$


In the absence of any information for a particular proposition, the trivial interval $[0,1]$ may be used. One important feature of this interpretation is that we assume the existence of an evaluation function. The notion of intervals represents our ignorance regarding the actual evaluation function.

It may also happen that one cannot determine the exact class of evaluation functions because of a lack of knowledge. That is, we may not assume the existence of a single-valued evaluation function. One may use a pair of evaluation functions that produce interval evaluations. Each of the evaluation functions may satisfy different axioms. For example, the lower evaluation is a belief function, while the upper evaluation is a plausibility function. Although intervals, in this case, can be regarded as constraints representing available information and knowledge, we may not explicitly express such constraints similar to equation (2).

The two interpretations of intervals can be related to different views of the theory of belief functions [12]. A pair of belief and plausibility functions produces an interval representation of uncertainty. There are at least two views for the interpretation of belief and plausibility functions. One view treats them as a pair of bounds that defines a family of probability functions [6], in a way similar to equation (2). Smets [13] referred to this interpretation as type-1 probabilistic reasoning, which is an upper and lower probability model. Our first interpretation of intervals corresponds to this view. In the type- 2 model referred to by Smets, no probability function is postulated to exist. Belief and plausibility functions are not considered as being bounds of a probability function, but as degrees of belief. Our second interpretation of intervals conforms to this view.

A key notion in interval based inference is the interval-valued truth. The inference process depends very much on the interpretation of such intervals. In order to deal with operations and interpretations of interval-valued truth, we present two methods in the subsequent sections, namely, interval computations and interval structures.

\section{Power Algebras and Interval Computations}

This section examines the notion of power algebras [1] and two special power algebras, one for numeric interval-valued truth and the other for non-numeric interval-valued truth.

\subsection{Power algebras}

Let $U$ be a set and o a binary operation on $U$. One can define a binary operation o $^{+}$on subsets of $U$ as follows $[1,18]$ :

$$
X \circ^{+} Y=\{x \circ y \mid x \in X, y \in Y\}
$$

for any $X, Y \subseteq U$. In general, one may lift any operation $f$ on elements of $U$ to an operation $f^{+}$on subsets of $U$, called the power operation of $f$. Suppose 
$f: U^{n} \longrightarrow U(n \geq 1)$ is an $n$-ary operation on $U$. The power operation $f^{+}$: $\left(2^{U}\right)^{n} \longrightarrow 2^{U}$ is defined by [1]:

$$
f^{+}\left(X_{0}, \ldots, X_{n-1}\right)=\left\{f\left(x_{0}, \ldots, x_{n-1}\right) \mid x_{i} \in X_{i} \text { for } i=0, \ldots, n-1\right\},
$$

for any $X_{0}, \ldots, X_{n-1} \subseteq U$. This provides a universal-algebraic construction approach. For any algebra $\left(U, f_{1}, \ldots, f_{k}\right)$ with base set $U$ and operations $f_{1}, \ldots, f_{k}$, its power algebra is given by $\left(2^{U}, f_{1}^{+}, \ldots, f_{k}^{+}\right)$.

The power operation $f^{+}$may carry some properties of $f$. For example, for a binary operation $f: U^{2} \longrightarrow U$, if $f$ is commutative and associative, $f^{+}$is commutative and associative, respectively. If $e$ is an identity for some operation $f$, the set $\{e\}$ is an identity for $f^{+}$. If an unary operation $f: U \longrightarrow U$ is an involution, i.e., $f(f(x))=f(x), f^{+}$is also an involution. On the other hand, many properties of $f$ are not carried over by $f^{+}$. For instance, if a binary operation $f$ is idempotent, i.e., $f(x, x)=x, f^{+}$may not be idempotent. If a binary operation $g$ is distributive over $f, g^{+}$may not be distributive over $f^{+}$.

A special type of power algebra is called interval algebra, in which operations on elements of $U$ are lifted to intervals of $U$, instead of arbitrary subsets of $U$. In doing so, the power operation $f^{+}$may carry additional properties of $f$. The notion of interval algebras forms a basis of uncertain reasoning with intervals. Two such interval algebras are examined in the following subsections.

\subsection{Interval number algebra}

An interval number $[\underline{a}, \bar{a}]$ with $\underline{a} \leq \bar{a}$ is the set of real numbers defined by:

$$
[\underline{a}, \bar{a}]=\{x \mid \underline{a} \leq x \leq \bar{a}\} .
$$

The set of all interval numbers is denoted by $I(\Re)$. Degenerate intervals of the form $[a, a]$ are equivalent to real numbers.

One can perform arithmetic operations on interval numbers by lifting arithmetic operations on real numbers [8]. Let $A$ and $B$ be two interval numbers, and let $*$ denote an arithmetic operation,,$+- \cdot$ or $/$ on pairs of real numbers. An arithmetic operation $*$ may be extended to pairs of interval numbers $A, B$ :

$$
A * B=\{x * y \mid x \in A, y \in B\} .
$$

The result $A * B$ is again a closed and bounded interval unless $0 \in B$ and the operation $*$ is division (in which case, $A * B$ is undefined). They can be computed by using formulas: for $A=[\underline{a}, \bar{a}]$ and $B=[\underline{b}, \bar{b}]$,

$$
\begin{aligned}
A+B & =[\underline{a}+\underline{b}, \bar{a}+\bar{b}], \\
A-B & =[\underline{a}-\bar{b}, \bar{a}-\underline{b}], \\
A \cdot B & =[\min (\underline{a} \underline{b}, \underline{a} \bar{b}, \bar{a} \underline{b}, \bar{a} \bar{b}), \max (\underline{a} \underline{b}, \underline{a} \bar{b}, \bar{a} \underline{b}, \bar{a} \bar{b})], \\
A / B & =[\underline{a}, \bar{a}] \cdot[1 / \bar{b}, 1 / \underline{b}], \quad 0 \notin[\underline{b}, \bar{b}] .
\end{aligned}
$$


In the special case where both $A$ and $B$ are positive intervals, the multiplication can be simplified to:

$$
A \cdot B=[\underline{a} \underline{b}, \bar{a} \bar{b}], \quad 0 \leq \underline{a} \leq \bar{a}, \quad 0 \leq \underline{b} \leq \bar{b} .
$$

One may lift any operations on real numbers, such as min and max, to power operations on intervals of real numbers [19]. Interval number algebra may serve as a basis for interval reasoning with numeric truth values, such as interval fuzzy reasoning [19], and interval probabilistic reasoning [11,16].

Example 5. Interval min-max fuzzy reasoning. Using the results from interval number algebra, we obtain the following inference rules for interval fuzzy reasoning [19]:

$$
\begin{array}{ll}
\text { (if2) } & {\left[f v_{*}(\neg \phi), f v^{*}(\neg \phi)\right]=\left[1-f v^{*}(\phi), 1-f v_{*}(\phi)\right],} \\
\text { (if3) } & {\left[f v_{*}(\phi \wedge \psi), f v^{*}(\phi \wedge \psi)\right]=\left[\min \left(f v_{*}(\phi), f v_{*}(\psi)\right), \min \left(f v^{*}(\phi), f v^{*}(\psi)\right)\right],} \\
\text { (if4) } & {\left[f v_{*}(\phi \vee \psi), f v^{*}(\phi \vee \psi)\right]=\left[\max \left(f v_{*}(\phi), f v_{*}(\psi)\right), \max \left(f v^{*}(\phi), f v^{*}(\psi)\right)\right] .}
\end{array}
$$

This is an interval extension of the system given in Example 2.

Example 6. Interval probabilistic reasoning. According to the laws of probability and interval number algebra, we have the following rules for interval probabilistic reasoning [16]:

$$
\begin{array}{ll}
\text { (ip2) } & {\left[P_{*}(\neg \phi), P^{*}(\neg \phi)\right]=\left[1-P^{*}(\phi), 1-P_{*}(\phi)\right],} \\
\text { (ip3) } & P_{*}(\phi \wedge \psi) \geq \max \left(0, P_{*}(\phi)+P_{*}(\psi)-P^{*}(\phi \vee \psi)\right), \\
& P^{*}(\phi \wedge \psi) \leq \min \left(P^{*}(\phi), P^{*}(\psi), P^{*}(\phi)+P^{*}(\psi)-P_{*}(\phi \vee \psi)\right), \\
\text { (ip4) } & P_{*}(\phi \vee \psi) \geq \max \left(P_{*}(\phi), P_{*}(\psi), P_{*}(\phi)+P_{*}(\psi)-P^{*}(\phi \wedge \psi)\right), \\
& P^{*}(\phi \vee \psi) \leq \min \left(1, P^{*}(\phi)+P^{*}(\psi)-P_{*}(\phi \vee \psi)\right)
\end{array}
$$

It is an interval extension of the system given in Example 3. Inference rules (ip3) and (ip4) cannot be expressed using equality as that of (if3) and (if4) in interval fuzzy reasoning. This stems from the fact that probabilistic logic is not truthfunctional.

\subsection{Interval set algebra}

Given two sets $A_{1}, A_{2} \in 2^{U}$ with $A_{1} \subseteq A_{2}$, the subset of $2^{U}$,

$$
\mathcal{A}=\left[A_{1}, A_{2}\right]=\left\{X \in 2^{U} \mid A_{1} \subseteq X \subseteq A_{2}\right\},
$$

is called a closed interval set [15]. The set $A_{1}$ is called the lower bound of the interval set and $A_{2}$ the upper bound. An interval set is a set of subsets bounded by two elements of the Boolean algebra $\left(2^{U}, \cap, \cup, \sim\right)$. Let $I\left(2^{U}\right)$ denote the set of all closed interval sets.

Let $\cap, \cup$ and - be set intersection, union and difference defined on $2^{U}$. We define the following binary operations on interval sets by lifting set-theoretic 
operations. For two interval sets $\mathcal{A}=\left[A_{1}, A_{2}\right]$ and $\mathcal{B}=\left[B_{1}, B_{2}\right]$, the interval set intersection, union and difference are respectively defined as:

$$
\begin{aligned}
& \mathcal{A} \sqcap \mathcal{B}=\{X \cap Y \mid X \in \mathcal{A}, Y \in \mathcal{B}\}, \\
& \mathcal{A} \sqcup \mathcal{B}=\{X \cup Y \mid X \in \mathcal{A}, Y \in \mathcal{B}\}, \\
& \mathcal{A} \backslash \mathcal{B}=\{X-Y \mid X \in \mathcal{A}, Y \in \mathcal{B}\} .
\end{aligned}
$$

The above defined operators are closed on $I\left(2^{U}\right)$, namely, $\mathcal{A} \sqcap \mathcal{B}, \mathcal{A} \sqcup \mathcal{B}$ and $\mathcal{A} \backslash \mathcal{B}$ are interval sets. In fact, these interval sets can be explicitly computed by:

$$
\begin{aligned}
& \mathcal{A} \sqcap \mathcal{B}=\left[A_{1} \cap B_{1}, A_{2} \cap B_{2}\right], \\
& \mathcal{A} \sqcup \mathcal{B}=\left[A_{1} \cup B_{1}, A_{2} \cup B_{2}\right], \\
& \mathcal{A} \backslash \mathcal{B}=\left[A_{1}-B_{2}, A_{2}-B_{1}\right] .
\end{aligned}
$$

The interval set complement $\neg\left[A_{1}, A_{2}\right]$ of $\left[A_{1}, A_{2}\right]$ is defined by $[U, U] \backslash\left[A_{1}, A_{2}\right]$. This is equivalent to $\left[U-A_{2}, U-A_{1}\right]=\left[\sim A_{2}, \sim A_{1}\right]$. Obviously, we have $\neg[\emptyset, \emptyset]=$ $[U, U]$ and $\neg[U, U]=[\emptyset, \emptyset]$. The system $\left(I\left(2^{U}\right), \sqcap, \sqcup\right)$ is a completely distributive lattice [9].

The set algebra $\left(2^{U}, \cap, \cup, \sim\right)$ is a special Boolean algebra. By using the same argument, one can lift operations in a Boolean algebra or a lattice [19]. Such interval algebras may be used for reasoning with interval extension of classical logic [17], and interval incidence calculus [20].

Example \%. A three-valued propositional logic. For the two-element Boolean al-

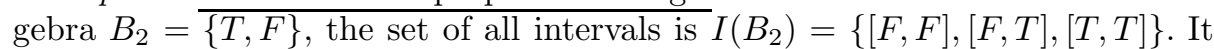
may be regarded as the truth values of a three-valued logic [19]. In this case, interval truth evaluation function $v_{*}, v^{*}$ from $L(\Phi)$ to $I\left(B_{2}\right)$ should satisfy the axioms:

(ic3) $\quad\left[v_{*}(\phi \wedge \psi), v^{*}(\phi \wedge \psi)\right]=\left[v_{*}(\phi) \wedge v_{*}(\psi), v^{*}(\phi) \wedge v^{*}(\psi)\right]$,

(ic3) $\quad\left[v_{*}(\phi \vee \psi), v^{*}(\phi \vee \psi)\right]=\left[v_{*}(\phi) \vee v_{*}(\psi), v^{*}(\phi) \vee v^{*}(\psi)\right]$

We therefore obtain a three-valued logic from the two-valued logic given in Example 1.

Example 8. Interval incidence calculus. The incidence calculus given in Example 4 can be extended to an interval version. In this case, the following axioms are used [17]:

(ii2) $\quad\left[i_{*}(\neg \phi), i^{*}(\neg \phi)\right]=\left[\sim i^{*}(\phi), \sim i_{*}(\phi)\right]$,

(ii3) $\quad\left[i_{*}(\phi \wedge \psi), i^{*}(\phi \wedge \psi)\right]=\left[i_{*}(\phi) \cap i_{*}(\psi), i^{*}(\phi) \cap i^{*}(\psi)\right]$,

(ii4) $\quad\left[i_{*}(\phi \vee \psi), i^{*}(\phi \vee \psi)\right]=\left[i_{*}(\phi) \cup i_{*}(\psi), i^{*}(\phi) \cup i^{*}(\psi)\right]$

Interval incidence calculus may be interpreted in term of the three-valued logic in Example 7. The set $i_{*}(\phi)$ may be interpreted as the subset of possible worlds in which the truth value of $\phi$ is $[T, T]$, while $i^{*}(\phi)$ is the subset of possible worlds in which the truth value of $\phi$ is $[T, T]$ or $[F, T]$. 


\section{Interval Structures}

The notion of interval structure was introduced by Wong, Wang and Yao [14] as a non-numeric representation of uncertainty. It may be considered as another interval extension of incidence calculus [3]. Let $W$ denote a set called possible worlds. An interval structure is a pair of mappings $\underline{i}, \bar{i}: L(\Phi) \longrightarrow 2^{W}$ satisfying the following axioms: for $\phi, \psi \in L(\Phi)$,

$$
\begin{array}{ll}
\text { (lp) } & \underline{i}(\phi)=\sim \bar{i}(\neg \phi), \quad \bar{i}(\phi)=\sim \underline{i}(\neg \phi), \\
\text { (11) } & \underline{i}(\perp)=\emptyset, \\
\text { (12) } & \underline{i}(\top)=W, \\
\text { (13) } & \underline{i}(\phi \wedge \psi)=\underline{i}(\phi) \cap \underline{i}(\psi), \\
\text { (u1) } & \bar{i}(\perp)=\emptyset, \\
\text { (u2) } & \bar{i}(\top)=W, \\
\text { (u3) } & \bar{i}(\phi \vee \psi)=\bar{i}(\phi) \cup \bar{i}(\psi) .
\end{array}
$$

The set $\underline{i}(\phi)$ may be interpreted as the subset of possible worlds in which $\phi$ is known or can be proved to be true, and $\sim \bar{i}(\phi)$ the subset of possible worlds in which $\phi$ is known or can be proved to be false. Interval structures may be regarded as an interval extension of incidence mappings. When $\underline{i}=\bar{i}$, the interval structure reduces to an incidence mapping.

For an interval structure $(\underline{i}, \bar{i})$, we have the property:

$$
\underline{i}(\phi) \subseteq \bar{i}(\phi),
$$

for any $\phi \in L(\Phi)$. They may be considered as the end points of the interval set:

$$
[\underline{i}(\phi), \bar{i}(\phi)]=\{X \mid \underline{i}(\phi) \subseteq X \subseteq \bar{i}(\phi)\} .
$$

The interval set corresponding to $(\underline{i}(\neg \phi), \bar{i}(\neg \phi))$ is:

$$
[\sim \bar{i}(\phi), \sim \underline{i}(\phi)]=\neg[\underline{i}(\phi), \bar{i}(\phi)] .
$$

Interval sets corresponding to $(\underline{i}(\phi \wedge \psi), \vec{i}(\phi \wedge \psi))$ and $(\underline{i}(\phi \vee \psi), \vec{i}(\phi \vee \psi))$ are:

$$
\begin{aligned}
& {[\underline{i}(\phi \wedge \psi), \bar{i}(\phi \wedge \psi)]=[\underline{i}(\phi) \cap \underline{i}(\psi), \bar{i}(\phi \wedge \psi)],} \\
& {[\underline{i}(\phi \vee \psi), \bar{i}(\phi \vee \psi)]=[\underline{i}(\phi \vee \psi), \bar{i}(\phi) \cup \bar{i}(\psi)] .}
\end{aligned}
$$

In general, they cannot be obtained from the interval set operations. Therefore, interval set algebra and interval structures offer two different approaches for interval reasoning.

Consider a special class of interval structures satisfying the axioms [20]:

$$
\begin{array}{ll}
\text { (14) } \quad \underline{i}(\phi) \subseteq \underline{i}(\psi) & \text { or } \quad \underline{i}(\psi) \subseteq \underline{i}(\phi), \\
\text { (u4) } \bar{i}(\phi) \subseteq \bar{i}(\psi) & \text { or } \quad \bar{i}(\psi) \subseteq \bar{i}(\phi),
\end{array}
$$


for any $\phi, \psi \in L(\Phi)$. We call such a class consonant interval structures. Axioms (14) and (u4) can be equivalently expressed as:

$$
\begin{array}{ll}
\text { (15) } \underline{i}(\phi \wedge \psi)=\underline{i}(\phi) & \text { or } \quad \underline{i}(\phi \wedge \psi)=\underline{i}(\psi), \\
(\mathrm{u} 5) \bar{i}(\phi \vee \psi)=\bar{i}(\phi) & \text { or } \bar{i}(\phi \vee \psi)=\bar{i}(\psi)
\end{array}
$$

In general, an interval structure does not have such properties.

The concept of incidence mappings is related to probabilistic reasoning. Let $P_{W}$ denote a probability function defined on $2^{W}$, and $i$ an incidence mapping from $L(\Phi)$ to $2^{W}$. The truth evaluation function defined by:

$$
P(\phi)=P_{W}(i(\phi))
$$

is a probability function defined on $L(\Phi)$. For an interval structure $(\underline{i}, \bar{i})$, a pair of truth evaluation functions defined by:

$$
\begin{aligned}
& \underline{P}(\phi)=P_{W}(\underline{i}(\phi)), \\
& \bar{P}(\phi)=P_{W}(\bar{i}(\phi)),
\end{aligned}
$$

is a pair of belief and plausibility functions [14]. Furthermore, if $(\underline{i}, \bar{i})$ is a consonant interval structure, $\underline{P}$ and $\bar{P}$ are a pair of necessity and possibility functions $[20]$.

Reasoning with interval-valued truth obtained from interval structures should follow the axioms of interval structures. It provides an approach different from and complementary to inference based on interval computations.

\section{Conclusion}

Interval based reasoning is a practical solution to problems in inference with incomplete, insufficient, or inconsistent information. Instead of using a singlevalued truth evaluation functions, one assigns each proposition with an intervalvalued truth. At least two views can be adopted for the interpretation of such intervals. Intervals may be interpreted as bounds of an unknown single-valued truth evaluation function. Alternatively, the end points of intervals can be interpreted as defining two truth evaluation functions, each is a single-valued evaluation function. With respect to such interpretations, we have presented a framework of interval reasoning. Two classes of interval reasoning approaches are introduced based on interval computations and interval structures. They are different from and complementary to each other.

\section{References}

1. C. Brink, Power structures, Algebra Universalis, 30, 177-216, 1993.

2. P.P. Bonissone, Summarizing and propagating uncertain information with triangular norms, International Journal of Approximate Reasoning, 1, 71-101, 1987. 
3. A. Bundy, Incidence calculus: a mechanism for probabilistic reasoning, Journal of Automated Reasoning, 1, 263-283, 1985.

4. D. Dubois, and P. Prade, Possibility Theory: an Approach to Computerized Processing of Uncertainty, Plenum Press, New York, 1988.

5. R. Fagin, and J.Y. Halpern, Uncertainty, belief, and probability, Computational Intelligence, 7, 160-173, 1991.

6. J.Y. Halpern, and R. Fagin, Two views of belief: belief as generalized probability and belief as evidence, Artificial Intelligence, 54: 275-317, 1992.

7. G.J. Klir, and B. Yuan, Fuzzy Sets and Fuzzy Logic: Theory and Applications, Prentice Hall, New Jersey, 1995.

8. R.E. Moore, Interval Analysis, Englewood Cliffs, New Jersey, Prentice-Hall, 1966.

9. C.V. Negoiţă, and D.A. Ralescu, Applications of Fuzzy Sets to Systems Analysis, Basel, Birkhäuser Verlag, 1975.

10. Z. Pawlak, Rough sets, International Journal of Computer and Information Sciences, 11, 341-356, 1982.

11. J.R. Quinlan, Inferno: a cautious approach to uncertain inference, The Computer Journal, 26, 255-269, 1983.

12. G. Shafer, A Mathematical Theory of Evidence, Princeton University Press, Princeton, 1976.

13. P. Smets, Resolving misunderstandings about belief functions, International Journal of Approximate Reasoning, 6, 321-344.

14. S.K.M. Wong, L.S. Wang, and Y.Y. Yao, On modeling uncertainty with interval structures, Computational Intelligence, 11, 406-426, 1995.

15. Y.Y. Yao, Interval-set algebra for qualitative knowledge representation, Proceedings of the Fifth International Conference on Computing and Information, 370-374, 1993.

16. Y.Y. Yao, A comparison of two interval-valued probabilistic reasoning methods, Proceedings of the 6th International Conference on Computing and Information, May 26-28, 1994, Peterborough, Ontario, Canada. Special issue of Journal of Computing and Information, 1, 1090-1105 (paper number D6), 1995.

17. Y.Y. Yao, and X. Li, Comparison of rough-set and interval-set models for uncertain reasoning, Fundamenta Informaticae, 27, 289-298, 1996.

18. Y.Y. Yao, and N. Noroozi, A unified framework for set-based computations, Proceedings of the 3rd International Workshop on Rough Sets and Soft Computing, Lin, T.Y. (Ed.), San Jose State University, 236-243, 1994.

19. Y.Y. Yao, and J. Wang, Interval based uncertain reasoning using fuzzy and rough sets, Advances in Machine Intelligence 83 Soft-Computing, Volume IV, Wang, P.P. (Ed.), Department of Electrical Engineering, Duke University, Durham, North Carolina, USA, 196-215, 1997

20. Y.Y. Yao, S.K.M. Wong, and L.S. Wang, A non-numeric approach to uncertain reasoning, International Journal of General Systems, 23, 343-359, 1995.

21. L.A. Zadeh, Fuzzy sets, Information \& Control, 8, 338-353, 1965. 